\title{
"CONCENTRATED CAPACITY" MODEL OF ION-EXCHANGE FUNNELING IN A MODIFIED (THIN FILM COATED) HETEROGENEOUS ELECTRODIALYSIS MEMBRANE
}

\author{
$\mathrm{BY}$ \\ STANislaV ANTONTSEV (Universidade da Beira Interior, Covilhã 6200, Portugal), \\ ANVARBEK M. MEIRMANOV (Universidade da Beira Interior, Covilhã 6200, Portugal), \\ ISAAK RUBINSTEIN (Blaustein Institute for Desert Research, Ben-Gurion University of the \\ Negev, Sede Boqer Campus 84990, Israel), \\ AND
}

BORIS ZALTZMAN (Blaustein Institute for Desert Research, Ben-Gurion University of the Negev, Sede Boqer Campus 84990, Israel)

\begin{abstract}
Inexpensive highly permselective heterogeneous ion exchange membranes are prohibitively polarizable by a direct electric current for use in electrodialysis. According to recent experiments, polarizability of these membranes may be considerably reduced by casting on their surface a thin layer of cross-linked polyelectrolyte, weakly charged with the same sign as the membrane's charge. The present paper is concerned with this effect. In order to explain this feature, a simple limiting ion-exchange 'funnel' model of a modified membrane is derived from the original two-layer model. In this model, asymptotically valid for a thin coating, solution of the ionic transport equations in it is replaced, via a suitable averaging procedure, by a single nonlinear boundary condition for the membrane/solution interface, which itself has the same order as the bulk equation. Rigorous analysis of the 'funnel' model shows that the value of the limiting current through a modified membrane, which is the main quantitative characteristic of its polarizability, is equal to that through a homogeneous membrane for any positive value of the funneling parameter. The limiting problem for a vanishing funneling parameter is analyzed.
\end{abstract}

1. Introduction. Electrodialysis is an ion separation (e.g., desalination) process based upon passing a direct electric current through an electrolyte solution flanked by very thin (a few hundred of microns thick) charge-selective (permselective) polymer films, known as ion exchange membranes. Two types of ion-exchange membranes are distinguished: homogeneous and heterogeneous ones.

Received January 4, 2002.

(C)2004 Brown University 
Homogeneous membranes, consisting of cross-linked polyelectrolyte, are prohibitively expensive for their practical use in a large scale desalination by electrodialysis. On the other hand, inexpensive heterogeneous membranes, made of very small ion exchange beads sealed in a neutral polymer matrix, is largely impaired by their prohibitively high polarizability compared to that of a homogeneous membrane. By this we mean that the differential resistance of a heterogeneous membrane with the adjacent electrolyte layers increases more rapidly with the increase of current than that of a homogeneous membrane. This increase is the essence of concentration polarization $(\mathrm{CP})$ in a solution layer adjacent to a charge-selective body (ion exchange membrane, electrode) under the passage of an electric current. The electrolyte concentration gradient which develops results in a typical nonlinear steady state voltage/current dependence, schematically depicted in Fig. 1. The following three regions are typically distinguishable in such a curve. The low current Ohmic region I is followed by a plateau with a much lower slope (region II, the limiting current). Inflection of the voltage-current curve at the plateau is followed by the 'over-limiting' region III. Transition to the over-limiting regime is accompanied by the appearance of low frequency excess electric noise. For a recent discussion of the over-limiting phenomena the interested reader is referred to [1]-[4]. In the current context, we are concerned with the classical 'under-limiting' CP regions I and II.

Higher polarizability of a heterogeneous membrane compared to a homogeneous one amounts to a lower limiting current through the former than through the latter. Yet, the recent experiments suggest that polarizability of heterogeneous membranes may be considerably reduced by casting on their surface a thin layer of a cross-linked polyelectrolyte (ion-exchange coating), weakly charged with the same sign as the membrane's charge [5].

In our previous study [4], we proposed a theoretical explanation of this phenomenon in terms of a model considering two layers: a homogeneous ion-exchange layer adjacent to the membrane surface and an electrolyte layer adjacent to it. Numerical solution of the respective boundary value problem (see [4]) shows that, indeed, even a very thin and lightly charged coating of this kind increases the value of the limiting current to that of a homogeneous membrane. To explain this feature, a relatively simple 'funnel' model of a modified membrane was derived from the original two-layer one. In this model, asymptotically valid for a thin coating, solution of the ionic transport equations in it is replaced, via a suitable averaging procedure, by a boundary condition of 'concentrated capacity' type for the membrane/solution interface. This nonlinear boundary condition contains derivatives of the same order as the 'bulk' equation.

In this paper we rigorously analyze the 'funnel' model. We show that the aforementioned property of the limiting current is an exact feature of this model, the underlying physical phenomenon being the 'funneling' of counterions by the charged layer from the impermeable parts of the membrane towards the 'entrance gates'. The paper is organized as follows. In Sec. 2 we briefly describe the results of [4], formulate the full two-layer model problem, and reproduce the formal asymptotic transition to the 'funnel' problem. In Sec. 3 we establish the existence and uniqueness of the smooth solution to this problem. Finally, in Sec. 4 we study the asymptotic behavior of the 'funnel' problem when 


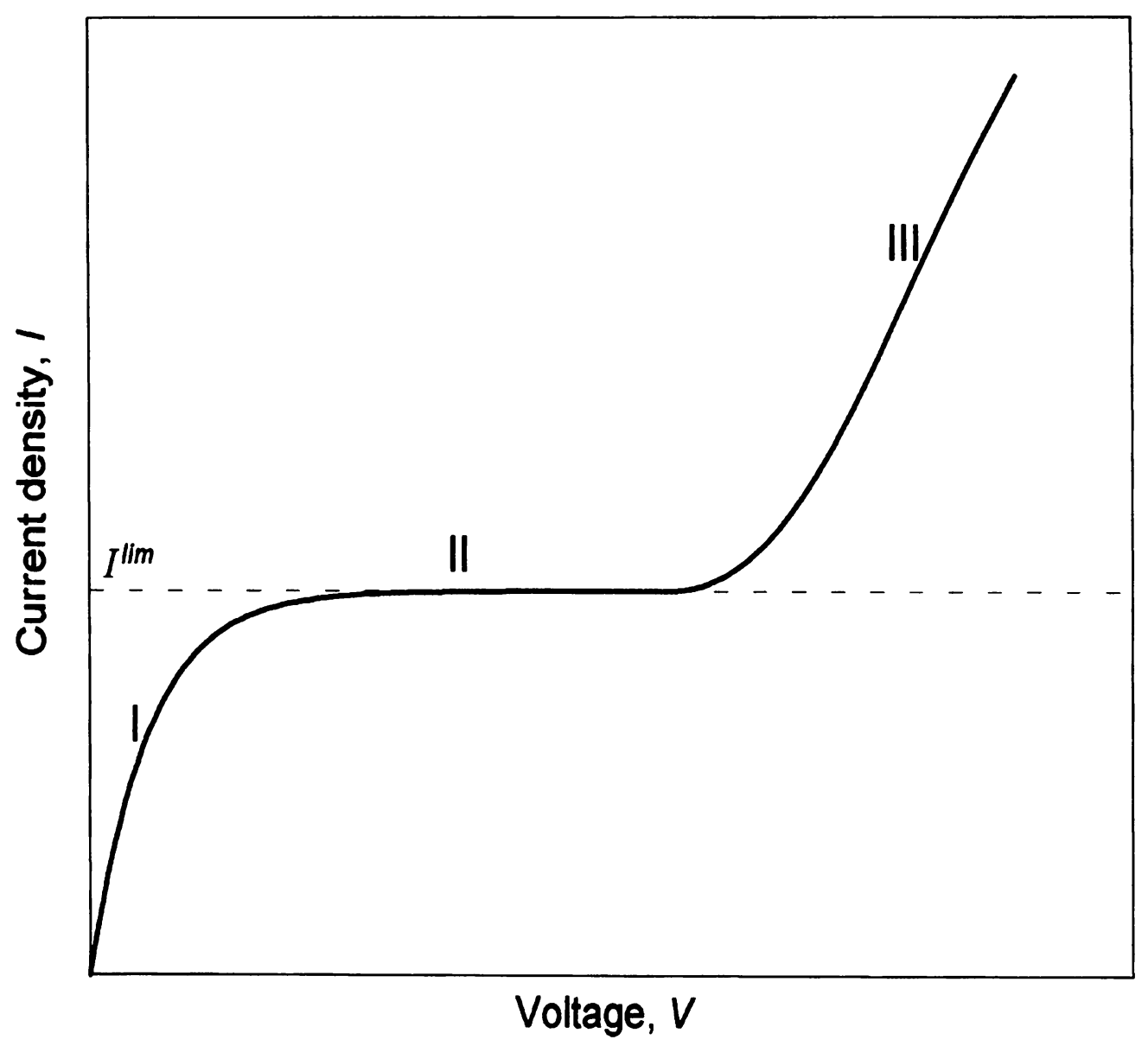

FIG. 1. Typical voltage/current dependence in electrodialysis

the control parameter (product of the dimensionless charge density in the coating to its thickness) tends to 0 . It is shown that the limit is a solution of a certain free boundary problem.

2. Two models of electrodiffusion in a two-dimensional cell at a modified heterogeneous membrane. Let us consider a diffusion layer of univalent electrolyte adjacent to a charged coating layer on a flat heterogeneous membrane. Confining ourself to a two-dimensional case, let us model the heterogeneous membrane/coating interface as a periodic array of conductive, ideally cation permselective strips and insulating strips. We direct the $x$ axis normal to the interface, with the origin at the outside, 'bulk' edge of the diffusion layer, and $y$ parallel to the interface and perpendicular to the strips at it. Thus, ion transfer in the diffusion layer and the coating may be reduced to that in a single periodicity cell confined by the membrane/coating interface, bulk edge of the 
diffusion layer, and the two symmetry planes $y=0,1$ (middle of the conductive and insulating strips, respectively).

In terms of natural dimensionless variables, the two-dimensional Nernst-Planck equations for locally electroneutral steady-state electrodiffusion of cat- and anions in the diffusion layer $(0<x<\delta, 0<y<1)$ (see $[6])$ are:

$$
\begin{aligned}
& \nabla(\nabla c+c \nabla \varphi)=0, \\
& \nabla(\nabla c-c \nabla \varphi)=0 .
\end{aligned}
$$

Here $c$ and $\varphi$ are the dimensionless electrolyte concentration and dimensionless electric potential, respectively.

By addition and subtraction, Eqs. (1), (2) are conveniently rewritten as the steady state diffusion (Laplace) and current continuity equations, respectively.

$$
\begin{aligned}
\Delta c & =0, \\
\nabla(c \nabla \varphi) & =0 .
\end{aligned}
$$

Similarly to Eqs. (1), (2), the electrodiffusion equations for the ion-exchange coating $(\delta<x<\delta+\varepsilon, 0<y<1)$ are

$$
\begin{aligned}
\nabla(\nabla p+p \nabla \varphi) & =0, \\
\nabla(\nabla n-n \nabla \varphi) & =0,
\end{aligned}
$$

where $p$ and $n$ are the cat- and an-ion concentrations, respectively, related by the local electroneutrality condition

$$
p=n+N,
$$

where $N>0$ is the fixed charge concentration in the cat- and coating, assumed hereon constant.

Continuity of the ionic fluxes and the electrochemical potentials at the coating/electrolyte interface $(x=\delta, 0<y<1)$ imply

$$
\begin{aligned}
\left.\left(p_{x}+p \varphi_{x}\right)\right|_{x=\delta+0} & =\left.\left(c_{x}+c \varphi_{x}\right)\right|_{x=\delta-0}, \\
\left.\left(n_{x}-n \varphi_{x}\right)\right|_{x=\delta+0} & =\left.\left(c_{x}-c \varphi_{x}\right)\right|_{x=\delta-0}, \\
\left.(\ln p+\varphi)\right|_{x=\delta+0} & =\left.(\ln c+\varphi)\right|_{x=\delta-0}, \\
\left.(\ln n-\varphi)\right|_{x=\delta+0} & =\left.(\ln c-\varphi)\right|_{x=\delta-0} .
\end{aligned}
$$

In Eqs. (8), (9), equal ionic diffusivities for the solution and the coating have been assumed for simplicity.

Simplest relevant boundary conditions at the heterogeneous membrane/coating interface $(x=\delta+\varepsilon, 0<y<1)$ pertain to the galvanostatic regime of operation, assuming a uniform distribution of current over the cation-permeable portion of the interface $(x=\delta+\varepsilon, 0<y<h)$. These boundary conditions read

$$
\begin{aligned}
& \left.\left(p_{x}+p \varphi_{x}\right)\right|_{x=\delta+\varepsilon}= \begin{cases}-i, & 0<y<h, \\
0, & h<y<1,\end{cases} \\
& \left.\left(n_{x}-n \varphi_{x}\right)\right|_{x=\delta+\varepsilon}=0 .
\end{aligned}
$$


Here $i$ is the constant dimensionless current density through the conductive strip of dimensionless half-width $h(I \stackrel{\text { def }}{=} i h$ is the average current density through the membrane). Boundary condition (13) pertains to impermeability for co-ions (anions) of both the insulating and conductive portion of the ideally cation permselective heterogeneous membrane under consideration. For the symmetry planes $y=0,1,0<x<\delta+\varepsilon$, we have

$$
\left.p_{y}\right|_{y=0,1}=\left.n_{y}\right|_{y=0,1}=\left.c_{y}\right|_{y=0,1}=\left.\varphi_{y}\right|_{y=0,1}=0 .
$$

Finally, the boundary conditions at the outer bulk edge of the diffusion layer $x=0$, $0<y<1$ are

$$
\begin{gathered}
c(0, y)=1, \\
\varphi(0, y)=0 .
\end{gathered}
$$

(The normalization condition (16) merely specifies the arbitrary constant in the definition of the electric potential.)

We point out first that the impermeability of the membrane for anions, Eq. (13), together with Eqs. (2), (6) implies

$$
\varphi(x, y)=\ln c(x, y) .
$$

Furthermore, for $\varepsilon=0$ (or $N=0$ ) the boundary value problem (3)-(17) is reduced to that for an unmodified heterogeneous membrane.

Next, we analyze the effect described in the Introduction, employing the fact that it takes place even for a very thin coating. More precisely, we derive from the full two-layer formulation (3)-(17) a simple limiting model, asymptotically valid for $\varepsilon \rightarrow 0, N \rightarrow \infty$, such that their product

$$
\beta \stackrel{\text { def }}{=} \varepsilon N=O(1)
$$

remains finite. For this purpose we first rewrite Eqs. (5), (6) in a more convenient form by using the standard definition [6]

$$
\sigma \stackrel{\text { def }}{=} p+n \text {. }
$$

Substitution of (19) into the sum of (5) and (6) yields, using (6), (7), and (17),

$$
\nabla\left(\nabla \sigma+N \frac{\nabla \sigma}{\sigma-N}\right)=0, \delta<x<\delta+\varepsilon, 0<y<1
$$

whereas the continuity conditions (8), (9) and (10), (11) yield, respectively,

$$
\begin{aligned}
\left.\left(\sigma_{x}+N \frac{\sigma_{x}}{\sigma-N}\right)\right|_{x=\delta+0} & =\left.2 c_{x}\right|_{x=\delta-0}, \\
\left(\sigma^{2}(\delta+0, y)-N^{2}\right) & =4 c^{2}(\delta-0, y) .
\end{aligned}
$$

On the other hand, substitution of (19) into the sum of boundary conditions (12) and (13) yields, using (7) and (17),

$$
\left.\left(\sigma_{x}+N \frac{\sigma_{x}}{\sigma-N}\right)\right|_{x=\delta+\varepsilon}= \begin{cases}-i, & 0<y<h \\ 0, & h<y<1\end{cases}
$$


Integration of Eq. (20) with respect to $x$ over the interval $\delta<x<\delta+\varepsilon$ yields, to the leading order in $\varepsilon$, using Eqs. (21)- $(23)^{1}$,

$$
\beta v_{y y}-\left.2 c_{x}\right|_{x=\delta}= \begin{cases}i, & 0<y<h \\ 0, & h<y<1\end{cases}
$$

Here, $\beta$ is defined by Eq. (18) and

$$
v(y) \stackrel{\text { def }}{=} \frac{\sigma(\delta, y)}{N}-1+\ln \left(\frac{\sigma(\delta, y)}{N}-1\right) .
$$

Eqs. (22), (25) yield

or

$$
\begin{aligned}
v(y) & =\sqrt{1+\frac{4}{N^{2}} c^{2}(\delta, y)}-1+\ln \left(\sqrt{1+\frac{4}{N^{2}} c^{2}(\delta, y)}-1\right) \\
& =\ln \frac{2}{N}+2 \ln c(\delta, y)+\frac{2}{N} c^{2}(\delta, y)+O\left(\frac{1}{N^{2}}\right)
\end{aligned}
$$

Substitution of Eq. (26) into Eq. (24) finally yields

$$
\beta\left(\frac{c_{y}(\delta, y)}{c(\delta, y)}\right)_{y}-c_{x}(\delta, y)= \begin{cases}i / 2, & 0<y<h \\ 0, & h<y<1\end{cases}
$$

Taking boundary condition (27) together with the Laplace equation (3) for $c(x, y)$, the symmetry conditions (14) and the boundary condition (15) form the limiting asymptotic ('funnel') model for the ionic transport in the diffusion layer at a modified heterogeneous membrane. In this model, the entire ionic transport in the coating is reduced to a single nonlinear boundary condition (27). For convenience we reproduce below the respective boundary value problem, which reads

$$
\begin{aligned}
\Delta c & =0,0<x<\delta, 0<y<1, \\
c(0, y) & =1,0<y<1, \\
\beta\left(\frac{c_{y}(\delta, y)}{c(\delta, y)}\right)_{y}-c_{x}(\delta, y) & =f(y) \stackrel{\text { def }}{=}\left\{\begin{array}{ll}
\frac{i}{2}, & 0<y<h, \\
0, & h<y<1,
\end{array}, 0<y<1,\right. \\
\left.c_{y}\right|_{y=0,1} & =0,0<x<\delta .
\end{aligned}
$$

${ }^{1}$ Eq. (24) is obtained using the relations

where

$$
\int_{\delta}^{\delta+\varepsilon} w(x, y) d x=\varepsilon v(y)+O\left(\varepsilon^{2}\right),
$$

$$
w=\frac{\sigma(x, y)}{N}-1+\ln \left(\frac{\sigma(x, y)}{N}-1\right) .
$$


In Sec. 3 we will rigorously analyze the boundary value problem (28)-(31) and show it to be well-posed, that is, to possess a unique classical solution. In this section, we will also show that for any finite value of the "funneling" parameter $\beta$, the value of the limiting current in the system is $2 / \delta$, that is, the value for a homogeneous membrane. This latter result is easily recovered by inspection. Indeed, the limiting current is that current value for which the lowest interface concentration at $x=\delta, y=0$ vanishes. On the other hand, by inspection, the flat one-dimensional concentration distribution,

$$
c^{\lim }(x, y)=1-\frac{x}{\delta}
$$

with an identically vanishing interface concentration at $x=\delta$, is a solution to (28)-(31) corresponding to the limiting current $I^{\mathrm{lim}} \stackrel{\text { def }}{=} i^{\mathrm{lim}} h=2 / \delta$. Given this result for any finite $\beta$, the natural question to be addressed is how does, for a vanishing $\beta$, the limiting current acquire its low 'heterogeneous' value? The answer to this question will be provided by the asymptotic analysis of the boundary value problem (28)--(31) for $\beta \rightarrow 0$, carried out in Sec. 4.

3. Well-posedness of the 'funnel' problem. We begin with consideration of problem (28)-(31) for current $I$ below the limiting value $I^{\mathrm{lim}}=2 / \delta$. To define a weak solution to this problem, we introduce the function $u(x, y) \stackrel{\text { def }}{=} \ln c(x, y)$. Then:

Definition. Nonpositive function $u(x, y) \in C^{1}(\bar{\Omega}), \Omega \stackrel{\text { def }}{=}(0, \delta) \times(0,1)$, is a weak solution to the problem (28)-(31), if the following integral equality

$$
\left.\int_{0}^{1}\left(\beta u_{y} \varphi_{y}\right)\right|_{x=\delta} d y+\int_{\Omega} e^{u}(\nabla u \nabla \varphi) d x d y+\left.\int_{0}^{1}(f \varphi)\right|_{x=\delta} d y=0
$$

holds for every function $\varphi(x, y) \in H^{1}(\Omega),\left.\varphi\right|_{x=0}=0$.

First, we show the uniqueness of the weak solution.

THEOREM 1. The weak solution to the problem (28)-(31) is unique.

Proof. Let us assume by way of contradiction the existence of two different weak solutions $u_{1}$ and $u_{2}$ and consider the difference $\bar{c}(x, y) \stackrel{\text { def }}{=} e^{u_{1}}-e^{u_{2}}$. For every test function $\varphi(x, y) \in H^{2}(\Omega)$, such that $\left.\varphi_{y}\right|_{y=0,1}=0$ and $\left.\varphi\right|_{x=0}=0$, the following integral equality

$$
\beta \int_{0}^{1}\left(\left.\left.a(x) \bar{c}\right|_{x=\delta} \varphi_{x x}\right|_{x=\delta}-\left.\left.\bar{c}\right|_{x=\delta} \varphi_{y}\right|_{x=\delta}\right) d x+\int_{\Omega} \bar{c} \Delta \varphi d x d y=0
$$

holds. Here $a(x) \stackrel{\text { def }}{=} e^{u_{*}(x)}$ and $u_{*}(x) \in\left(u_{1}(x), u_{2}(x)\right)$.

To determine a test function $\varphi$ we consider the following boundary value problem

$$
\begin{cases}\Delta H=\Phi_{y}, & (x, y) \in \Omega, \\ a H_{x}+H=a \Phi, & x=\delta, \\ H_{y}=0, & y=0,1, \\ H_{x}=0, & x=0 .\end{cases}
$$

This problem has a unique solution for every function $\Phi(x, y) \in C^{1}(\bar{\Omega})$. 
Taking $\Phi(x, y)$, such that $\Phi(0, y)=0$, we find that the function $\varphi$,

$$
\varphi(x, y)=\int_{0}^{x} H(\xi, y) d \xi
$$

is a solution to the following boundary value problem:

$$
\begin{cases}\Delta \varphi=\Phi, & (x, y) \in \Omega, \\ a \varphi_{y y}-\varphi_{x}=0, & x=\delta, \\ \varphi_{y}=0, & y=0,1, \\ \varphi=0, & x=0 .\end{cases}
$$

Substituting this function into the equality (34) we obtain that

$$
\int_{\Omega} \bar{c} \Phi d x d y=0
$$

for every smooth function $\Phi(x, y)$ vanishing at $x=0$. Taking $\Phi=\bar{c}$ we find that

$$
\int_{\Omega} \bar{c}^{2} d x d y=0
$$

and, thus, $\bar{c}=0$ in $\Omega$. The obtained contradiction completes the proof.

TheOREm 2. There exists a weak solution to the problem (28)-(31) for any $0<I<2 / \delta$. This solution is infinitely many times differentiable for all $(x, y)$ except the point $(\delta, h)$ and thus, this is a classical solution too.

Proof. The proof is based on the use of the Leray-Shauder Theorem. Let us define the set $L$ :

$$
L \stackrel{\text { def }}{=}\left\{v \in C^{1, \alpha}(\bar{\Omega}),-M_{0} \leq v \leq 0,\left\|v_{0}\right\|_{\Omega}^{(1, \alpha)} \leq M_{1}\right\} .
$$

The constants $M_{0}, M_{1}$ will be defined below, $0<\alpha<1 / 2$.

Let us begin with consideration of a modified 'funnel' problem (28)-(31) with function $f$ in the equation (30) replaced by a smooth function $f_{\varepsilon}(y), \varepsilon>0$ :

$$
f_{\varepsilon}(y) \in C^{\infty}[0,1], \lim _{\varepsilon \rightarrow 0} f_{\varepsilon}(y)=f(y) \text { for all } y \neq h, \int_{0}^{1} f_{\varepsilon}(y) d y=I / 2 .
$$

For every fixed $\varepsilon>0$ we define the operator $F_{\lambda}: v \rightarrow u, \lambda \in[0,1]$, where $u(x, y)$ is a solution of the following linear problem:

$$
\begin{gathered}
\int_{\Omega} e^{v} \nabla u \nabla \varphi d x d y+\left.\left.\beta \int_{0}^{1} u_{y}\right|_{x=\delta} \varphi_{y}\right|_{x=\delta} d y=-\left.\lambda \int_{0}^{1} f_{\varepsilon} \varphi\right|_{x=\delta} d y \\
u_{y}=0, y=0,1 \\
u=0, x=0 .
\end{gathered}
$$

The left-hand side of Eq. (39) defines a norm in the space of functions from $H^{1}(\Omega) \cap$ $H^{1}(\Gamma), \Gamma \stackrel{\text { def }}{=}\{x=\delta, 0<y<1\}$, vanishing at $x=0$ and periodic in $y$. The right-hand side of this equation is a bounded linear functional in this space. Thus, applying the Riesz Theorem on the representation of a linear functional in the Hölder space, we obtain that there exists a unique solution to the problem (39)-(41), $u(x, y) \in H^{1}(\Omega) \cap H^{1}(\Gamma)$. 
Since $u(x, y)$ is a weak solution to the following equation

$$
\Delta u+\nabla u \nabla v=0 \text { in } \Omega,
$$

in the sense of integral equality (39), the boundedness of the derivative $u_{y}(\delta, y)$ in the space $L^{2}(0,1)$ yields

$$
u_{x}(\delta, y) \in L^{2}(0,1)
$$

Using this regularity of the normal derivative, we obtain that $u(x, y)$ is a weak solution of Eq. (42), satisfying the Neumann boundary condition at $x=\delta$ in the following sense:

$$
\int_{\Omega} e^{v} \nabla u \nabla \varphi d x d y=\left.\left.\int_{0}^{1} e^{v} u_{x}\right|_{x=\delta} \varphi\right|_{x=\delta} d y
$$

Combining Eqs. (39) and (44), we find that $u(\delta, y)$ is a weak solution to the following equation:

$$
-\beta\left(\left.u\right|_{x=\delta}\right)_{y y}+\left.e^{v} u_{x}\right|_{x=\delta}=-\lambda f_{\varepsilon}(y), y \in(0,1) .
$$

This equation together with the condition (43) yield

$$
\left(\left.u\right|_{x=\delta}\right)_{y y} \in L^{2}(0,1)
$$

and, consequently,

$$
\left.u\right|_{x=\delta} \in C^{1, \tilde{\alpha}}[0,1] \text { for all } \tilde{\alpha} \in(0,1 / 2) .
$$

Applying the classical results of the theory of elliptic equations, we conclude that

$$
u(x, y) \in C^{1, \tilde{\alpha}}(\bar{\Omega}) .
$$

Using the last estimate, we conclude that $u(x, y)$ is a classical $C^{2, \alpha}(\bar{\Omega})$-smooth solution of the following boundary value problem:

$$
\begin{cases}-\beta\left(\left.u\right|_{x=\delta}\right)_{y y}+\left.e^{v} u_{x}\right|_{x=\delta}=-\lambda f_{\varepsilon}(y), & y \in(0,1) \\ \Delta u+\nabla u \nabla v=0, & (x, y) \in \Omega, \\ u_{y}=0, & x \in[0, \delta], y=0,1, \\ u=0, & x=0, y \in[0,1] .\end{cases}
$$

Therefore, the operator $F_{\lambda}$ maps the set $L$ into $C^{2, \alpha}(\bar{\Omega})$. Continuity of this operator follows from the continuous dependence of the solution to the problem (48) on its coefficients. Namely, if $u_{1}=F_{\lambda}\left(v_{1}\right), u_{2}=F_{\lambda}\left(v_{2}\right), \bar{u}=u_{1}-u_{2}, \bar{v}=v_{1}-v_{2}$, taking the difference of the respective integral equalities (39) for $u_{2}$ and $u_{1}$, and substituting $\varphi=\bar{u}$ into this difference, we obtain

$$
\int_{\Omega} e^{v_{1}}(\nabla \bar{u})^{2} d x d y+\beta \int_{0}^{1}\left(\left.\bar{u}_{y}\right|_{x=\delta}\right)^{2} d y=\int_{\Omega}\left(e^{v_{1}}-e^{v_{2}}\right)(\nabla \bar{u})^{2} d x d y .
$$

Since $v_{1}, v_{2} \in L$, the last equality yields

$$
\int_{\Omega}(\nabla \bar{u})^{2} d x d y+\beta \int_{0}^{1}\left(\left.\bar{u}_{y}\right|_{x=\delta}\right)^{2} d y \leq K_{1} \int_{\Omega} \bar{v}^{2} d x d y
$$

where $K_{1} \stackrel{\text { def }}{=} e^{2 M_{0}} \max \left(\left|\nabla u_{1}\right|+\left|\nabla u_{2}\right|\right)^{2}$, and, thus,

$$
\|\bar{u}\|_{C^{1, \alpha}(\bar{\Omega})} \leq K_{2}\|\bar{v}\|_{H^{2}(\Omega)} .
$$


(To obtain (51) we used the respective interpolation inequality (see [7]), in which the norm in the space $C^{1, \alpha}$ is estimated by the norm in the embedded space $C^{2, \alpha}$ and the norm in the embedding space $H^{1}$.) The latter estimate yields the continuity of the operator $F_{\lambda}$ in $v$.

Similarly, considering the difference $\bar{u} \stackrel{\text { def }}{=} F_{\lambda_{1}}(v)-F_{\lambda_{2}}(v)$, we obtain uniform continuity of the operators $F_{\lambda}$ in $\lambda$ for a fixed function $v$.

Let us consider $\lambda=0$. Since $F_{0} v=0$ for all $v \in L$, the equation $u=F_{0} u$ has a unique solution $u \equiv 0$ and the map $G_{0}: v \rightarrow v-F_{0} v \equiv v$ is bijective.

The last condition of the Leray-Shauder Theorem which we have to check is the nonexistence of a fixed point of the map $F_{\lambda}$ on the boundary of the set $L$. Let us consider the fixed point $u=F_{\lambda} u$. Then, $u \in C^{2, \alpha}(\bar{\Omega})$ and, since $u(x, y)$ is a classical solution of the boundary value problem (48) with $v=u$, we observe that $u \in C^{\infty}(\bar{\Omega})$. Applying the maximum principle to the problem (48) we obtain that

$$
u(x, y) \leq 0 \text { in } \bar{\Omega} .
$$

Similarly, considering the respective boundary value problem on $u_{x}(x, y)$, using the maximum principle, we deduce that

$$
u_{x}(x, y) \leq 0 \text { in } \bar{\Omega} .
$$

Defining the function $w(y)$ as follows:

$$
w(x) \stackrel{\text { def }}{=} \int_{0}^{1} e^{u(x, y)} d y,
$$

and integrating Eq. (28) in $y$, we find that

$$
\begin{cases}w^{\prime \prime}(x)=0, & 0<x<\delta, \\ w(0)=1, & \\ w^{\prime}(\delta)=-\int_{0}^{1} f_{\varepsilon}(y) d y=-\frac{I}{2} . & \end{cases}
$$

The solution to the problem (54) reads

$$
w(x)=1-\frac{I}{2} x, 0 \leq x \leq \delta .
$$

Let us consider the boundary condition at $x=\delta$ in terms of $c(x, y)=e^{u(x, y)}$ :

$$
\beta(\ln c)_{y y}=f_{\varepsilon}(y)+c_{x} \text { on } x=\delta .
$$

Integrating this equation in $y$, we find that

$$
\beta(\ln c)_{y}=\int_{0}^{y} f_{\varepsilon} d s+\left.\int_{0}^{y} c_{x}\right|_{x=\delta} d s \text { on } x=\delta .
$$

Using the estimate (53) and the equality (56), we obtain that

$$
\beta(\ln c)_{y} \leq \int_{0}^{y} f_{\varepsilon} d s \leq \frac{I}{2} \text { on } x=\delta .
$$

The inequalities $f_{\varepsilon}(y) \geq 0,\left.\int_{0}^{y} c_{x}\right|_{x=\delta} d s \geq\left.\int_{0}^{1} c_{x}\right|_{x=\delta} d s \geq-\frac{I}{2}$, and Eq. (56) yield

$$
\beta(\ln c)_{y} \geq-\frac{I}{2} \text { on } x=\delta \text {. }
$$


Combining the estimates (57) and (58) we obtain that

$$
\beta\left|\frac{c_{y}}{c}\right| \leq \frac{I}{2} \text { on } x=\delta
$$

and, using the estimate (52), we find that

$$
\beta\left|u_{y}\right| \leq \frac{I}{2} \text { on } x=\delta
$$

By the mean-value theorem

$$
w(x)=\int_{0}^{1} e^{u(x, y)} d y=e^{u\left(x, y_{0}(x)\right)}
$$

and, thus,

$$
u\left(x, y_{0}(x)\right)=\ln w(x)
$$

Since

$$
u(x, y)=u\left(x, y_{0}(x)\right)+\int_{y_{0}}^{y} u_{y}(x, s) d s,
$$

using the estimate (59) together with Eq. (60), we obtain the estimate

$$
0 \geq u(x, y) \geq \ln w(x)-\frac{I}{2 \beta} \geq \ln w(0)-\frac{I}{2 \beta}=\ln \left(1-\frac{I \delta}{2}\right)-\frac{I}{2 \beta} .
$$

By defining the constant $M_{0}$ in (37), (50) as

$$
M_{0}=-2\left[\ln \left(1-\frac{I \delta}{2}\right)-\frac{I}{2 \beta}\right]
$$

we deduce that

$$
0 \geq u(x, y) \geq-\frac{M_{0}}{2} .
$$

Substituting $\varphi=u$ into Eq. (39) yields the estimate

$$
\int_{\Omega} e^{v}(\nabla u)^{2} d x d y+\beta \int_{0}^{1}\left(\left.u_{y}\right|_{x=\delta}\right)^{2} d y \leq \frac{I}{2} \max |u| \leq \frac{M_{0} I}{4} .
$$

Since $u$ is a solution to the boundary value problem (48) with $v=u$, using the estimate (63), we obtain that $\left\|\left.u_{x}\right|_{x=\delta}\right\|_{L^{2}(0,1)} \leq K_{3}$ for some positive constant $K_{3}$ and, applying this estimate in the boundary condition at $x=\delta$, we find that

$$
\left\|\left.u_{x x}\right|_{x=\delta}\right\|_{L^{2}(0,1)} \leq K_{4},
$$

for some positive constant $K_{4}$. Using the embedding of the space $H^{2}(0,1)$ into the space $C^{1, \alpha}[0,1], 0<\alpha<1 / 2$, we obtain

$$
\left\|\left.u\right|_{x=\delta}\right\|_{C^{1, \alpha}[0,1]} \leq K_{5}
$$

for some positive constant $K_{5}$ and

$$
\|u\|_{C^{1, \alpha}(\bar{\Omega})} \leq K_{6}
$$

for some positive constant $K_{6}$. Finally, defining the constant $M_{1}$ in (37) as $M_{1} \stackrel{\text { def }}{=} 2 K_{6}$, we establish the estimate

$$
\left\|\left.u\right|_{x=\delta}\right\|_{C^{1, \alpha}(\bar{\Omega})} \leq \frac{M_{1}}{2}
$$


Estimates (62) and (65) yield the non-existence of a fixed point of the map $F_{\lambda}$ on the boundary of the set $L$.

Thus, all the conditions of the Leray-Shauder Theorem hold and, therefore, the equation $u=F_{\lambda} u$ has a solution in the set $L$ for all $\lambda \in[0,1]$ and, in particular, for $\lambda=1$. Since the constant $M_{1}$ on the right-hand side of the estimate (65) depends only on the norm of the function $f_{\varepsilon}$ in the space $L^{2}(0,1)$, we obtain, taking the limit $\varepsilon \rightarrow 0$, that there exists a solution of the original problem $u(x, y) \in L$. Using the general theory of elliptic equations, we also find that $u \in C^{\infty}$ everywhere in $\bar{\Omega}$ except the point $(\delta, h)$. Therefore, $c(x, y)=e^{u(x, y)}$ is a classical solution of the problem (28)-(31).

Corollary. As the current $I$ approaches its limiting value $I^{\mathrm{lim}}=2 / \delta$, the solution $c(x, y)$ tends to the one-dimensional limiting solution $c^{\lim }(x, y)=1-x / \delta$ weakly in the space $L^{2}(\Omega)$.

Proof. Since the function $u(x, y)$ is nonpositive uniformly in $I$, the solution $c(x, y)=$ $e^{u(x, y)}$ is bounded uniformly in $I$. The following integral equation,

$$
\int_{\Omega} c \Delta \varphi d x d y-\int_{0}^{1} \varphi_{x}(0, y) d y+\int_{0}^{1} c(\delta, y) \varphi_{x}(\delta, y) d y=0,
$$

holds for any test function $\varphi(x, y) \in C^{2}(\Omega)$, such that $\left.\varphi\right|_{x=0, \delta}=0,\left.\varphi_{y}\right|_{y=0,1}=0$.

Integration of $c$ with respect to $y$ yields

$$
\int_{0}^{1} c(\delta, y) d y=w(\delta)=1-\frac{I \delta}{2}
$$

and, substituting this equality into Eq. (66), we find that

$$
\left|\int_{\Omega} c \Delta \varphi d x d y-\int_{0}^{1} \varphi_{x}(0, y) d y\right| \leq \frac{2}{\delta}\left(I^{\mathrm{lim}}-I\right) \max \left|\varphi_{x}\right| .
$$

Taking the limit $I \rightarrow I^{\text {lim }}$ in the estimate (67) and referring to the uniform in $I$ boundedness of $c(x, y)$, we obtain that $c(x, y) \rightarrow c^{\text {lim }}(x, y)$ weakly in $L^{2}(\Omega)$, where $c^{\lim }(x, y)$ is a weak solution to the following boundary value problem $\beta$ :

$$
\begin{cases}\Delta c^{\lim }=0, & (x, y) \in \Omega, \\ c^{\lim }=0, & x=\delta, \\ c^{\lim }=1, & x=0, \\ c_{y}^{\lim }=0, & y=0,1 .\end{cases}
$$

Problem (68) possesses a unique solution $c^{\lim }(x, y)=1-x / \delta$.

4. Asymptotic behavior for vanishing 'funnel' parameter $(\beta \rightarrow 0)$. In this section we consider a sequence of solutions to the problem (28)-(31) corresponding to different values of the control parameter $\beta>0$. Let us denote the respective solution by 
$C^{(\beta)}(x, y)$ :

$$
\begin{gathered}
\Delta C^{(\beta)}=0,(x, y) \in \Omega=(0, \delta) \times(0,1) \\
\beta\left(\ln C^{(\beta)}\right)_{y y}-C_{x}^{(\beta)}=f, \text { at } x=\delta, \\
C_{y}^{(\beta)}=0, \text { at } y=0, y=1, \\
C^{(\beta)}=1, \text { at } x=0 .
\end{gathered}
$$

Here

$$
f(y)=\left\{\begin{array}{l}
\frac{i}{2}>0,0 \leq y<h \\
0, h<y \leq 1
\end{array}\right.
$$

We begin with consideration of a regularized solution $C_{\varepsilon}^{(\beta)}, \varepsilon>0$, to the problem (69)(73) with a smooth right-hand side in the boundary condition $(70) f^{\varepsilon}(y) \in C^{\infty}[0,1]$,

$$
\begin{gathered}
f^{\varepsilon}(y)= \begin{cases}\frac{i}{2}, & 0 \leq y<h-\varepsilon, \\
0, & h+\varepsilon \leq y \leq 1,\end{cases} \\
\frac{d f^{\varepsilon}(y)}{d y} \leq 0 \text { and } \int_{0}^{1} f^{\varepsilon}(y) d y=\frac{I}{2}=\frac{i h}{2}, 0 \leq f^{\varepsilon} \leq \frac{i}{2} .
\end{gathered}
$$

LEMmA 3. There exists a unique classical solution $C_{\varepsilon}^{(\beta)}(x, y)$ to the regularized problem (69)-(72), (74) for any $0<I<2 / \delta$ and

$$
\begin{gathered}
0<\nu(\beta) \leq C_{\varepsilon}^{(\beta)}(x, y) \leq 1 \text { for all }(x, y) \in \Omega=(0, \delta) \times(0,1) \\
\frac{\partial}{\partial x} C_{\varepsilon}^{(\beta)}(x, y) \leq 0 \text { for all }(x, y) \in \Omega=(0, \delta) \times(0,1) \\
\int_{0}^{1} C_{\varepsilon}^{(\beta)}(\delta, y) d y=1-\frac{I \delta}{2} .
\end{gathered}
$$

Moreover

$$
\iint_{\Omega}\left|\nabla C_{\varepsilon}^{(\beta)}\right|^{2} d x d y+\left.\beta \int_{0}^{1} \frac{\left|C_{\varepsilon y}^{(\beta)}\right|^{2}}{C_{\varepsilon}^{(\beta)}}\right|_{x=\delta} d y \leq \frac{I \delta}{2}
$$

and

$$
\begin{gathered}
\iint_{\Omega}\left\{\left|C_{\varepsilon x x}^{(\beta)}\right|^{2}+\left|C_{\varepsilon x y}^{(\beta)}\right|^{2}+\left|C_{\varepsilon y y}^{(\beta)}\right|^{2}\right\} d x d y \\
+\left.\beta \int_{0}^{1}\left\{\frac{\left|C_{\varepsilon y y}^{(\beta)}\right|^{2}}{C_{\varepsilon}^{(\beta)}}+\frac{\left|C_{\varepsilon y}^{(\beta)}\right|^{4}}{\left(C_{\varepsilon}^{(\beta)}\right)^{3}}\right\}\right|_{x=\delta} d y \leq K_{1}(\varepsilon) .
\end{gathered}
$$

Proof. The first part of this Lemma was proved in the previous section (see Theorems 1 and 2). To complete the proof we need to obtain the estimates (79), (80). Multiplication of Eq. (69) by the function $C_{\varepsilon}^{(\beta)}$, integration over the domain $\Omega$ followed by integration by parts yield the estimate (79). Similarly, multiplying Eq. (69) by the function $C_{\varepsilon y y}^{(\beta)}$, integrating the obtained equality over the domain $\Omega$ and using integration by parts, we obtain the estimate $(80)$.

Thus, for any $\beta>0$,

$$
\left\|C_{\varepsilon}^{(\beta)}\right\|_{H^{2}(\Omega)} \leq K_{1}(\varepsilon),
$$


with constant $K_{1}(\varepsilon)$ depending on $\varepsilon$ and independent of $\beta$, and we may choose the subsequence $\beta_{n} \rightarrow 0$ such that $C_{\varepsilon}^{\left(\beta_{n}\right)}$ converge weakly in $H(\Omega)$ and strongly in

$$
W_{p}^{1}(\Omega) \cap W_{p}^{1}(\Gamma) \cap C^{0, \alpha}(\bar{\Omega}),(\text { for any } p>1, \alpha \in(0,1)),
$$

to some function $C_{\varepsilon}(x, y)$, such that

$$
C_{\varepsilon} \in W_{p}^{1}(\Omega) \cap W_{p}^{1}(\Gamma) \cap C^{\alpha}(\bar{\Omega}) .
$$

For convenience of presentation we will omit below the subscript $n$ and write $\beta \rightarrow 0$ instead of $\beta_{n} \rightarrow 0$. Let us prove that

$$
C_{\varepsilon x}^{(\beta)}+f^{\varepsilon} \rightarrow C_{\varepsilon x}+f^{\varepsilon} \text { strongly in } L_{p}(\Gamma)
$$

and

$$
\beta C_{\varepsilon y y}^{(\beta)}-\beta \frac{\left|C_{\varepsilon y}^{(\beta)}\right|^{2}}{C^{(\beta)}} \rightarrow 0 \text { strongly in } L_{1}(\Gamma)
$$

when $\beta \rightarrow 0$. Considering the following integrals

$$
\begin{gathered}
J_{2}^{(\beta)} \stackrel{\text { def }}{\equiv} \beta \int_{\Gamma}\left|C_{\varepsilon y y}^{(\beta)}\right| d y \leq \beta^{2-\mu} \int_{\Gamma} \frac{\left|C_{\varepsilon y y}^{(\beta)}\right|^{2}}{C_{\varepsilon}^{(\beta)}} d y+\beta^{\mu} \int_{\Gamma} C_{\varepsilon}^{(\beta)} d y, \mu \in(0,1), \\
J_{1}^{(\beta)} \stackrel{\text { def }}{\equiv} \beta \int_{\Gamma} \frac{\left|C_{\varepsilon y}^{(\beta)}\right|^{2}}{C_{\varepsilon}^{(\beta)}}\left|C_{\varepsilon y y}^{(\beta)}\right| d y \leq \beta^{2-\mu} \int_{\Gamma} \frac{\left|C_{\varepsilon y}^{(\beta)}\right|^{4}}{\left(C_{\varepsilon}^{(\beta)}\right)^{3}} d y+\beta^{\mu} \int_{\Gamma} C_{\varepsilon}^{(\beta)} d y,
\end{gathered}
$$

and using the estimates (79), (80), we find that $J_{1}^{(\beta)}, J_{2}^{(\beta)} \rightarrow 0$ as $\beta \rightarrow 0$. Taking the limit $\beta \rightarrow 0$ in the boundary condition (70) rewritten in the following form:

$$
\beta C_{\varepsilon x x}^{(\beta)}-\beta \frac{\left|C_{\varepsilon x}^{(\beta)}\right|^{2}}{C_{\varepsilon}^{(\beta)}}=C_{\varepsilon}^{(\beta)}\left(C_{\varepsilon x}^{(\beta)}+f_{\varepsilon}\right),
$$

we obtain that

$$
C_{\varepsilon}\left(C_{\varepsilon x}+f_{\varepsilon}\right)=0 \text { at } x=\delta .
$$

Taking the limit $\beta \rightarrow 0$ in the equations (69), (71), and (72), we find that

$$
C_{\varepsilon}(x, y) \in H^{2}(\Omega)
$$

and the limiting function $C_{\varepsilon}(x, y)$ satisfies the equations (69), (71), (72), and (78).

TheOREM 4. The limiting function $C_{\varepsilon}$ is Hölder continuous:

$$
C_{\varepsilon} \in C^{0, \alpha}(\bar{\Omega}),\left\|C_{\varepsilon}\right\|_{C^{0, \alpha}(\bar{\Omega})} \leq K_{6}, 0<\alpha<\frac{1}{3},
$$

uniformly in $\varepsilon>0$ and

$$
C_{\varepsilon} \in C^{1, \alpha}(\bar{\Omega}),\left\|C_{\varepsilon}\right\|_{C^{1, \alpha}(\bar{\Omega})} \leq K_{7}(\varepsilon) .
$$

The following monotonicities hold:

$$
\begin{aligned}
& \frac{\partial C_{\varepsilon}(x, y)}{\partial y} \geq 0 \text { in } \Omega \\
& \frac{\partial C_{\varepsilon}(x, y)}{\partial x} \leq 0 \text { in } \Omega .
\end{aligned}
$$


Proof. Rewriting condition (70) in the following form:

$$
\beta C_{\varepsilon x}^{(\beta)}=C_{\varepsilon}^{(\beta)}\left(\int_{0}^{y} C_{\varepsilon x}^{(\beta)}(\delta, s) d s+F(y)\right), F(y)=\int_{0}^{y} f(s) d s,
$$

and taking the limit $\beta \rightarrow 0$, we obtain

$$
C_{\varepsilon}\left(\int_{0}^{y} C_{\varepsilon x}(\delta, s) d s+F(y)\right)=0, \text { at } x=\delta .
$$

Let us define a harmonic function $\omega(x, y)$ as follows:

$$
\omega(x, y) \stackrel{\text { def }}{=} \int_{0}^{y} C_{\varepsilon x}(x, s) d s .
$$

Since it is conjugate to the harmonic function $C_{\varepsilon}(x, y)$ :

$$
\omega_{x}=C_{\varepsilon y}, \omega_{y}=-C_{\varepsilon x},
$$

the product $\omega(x, y) C_{\varepsilon}(x, y)$ is also harmonic:

$$
\Delta\left(\omega C_{\varepsilon}\right)=0 \text { in } \Omega .
$$

Let $\psi$ be a harmonic function such that

$$
\begin{gathered}
\psi=F \text { at } x=\delta, \\
\psi_{y}=0 \text { at } y=0,1,
\end{gathered}
$$

and

$$
\psi=0 \text { at } x=0 .
$$

Since $F \in W_{p}^{1}(\Gamma)$ for all $p>1$, then

$$
\psi \in W_{p}^{1}(\Omega) \cap W_{p}^{1}(\Gamma) \text { for any } p>1
$$

and

$$
\|\psi\|_{W_{p}^{1}(\Omega)}+\|\psi\|_{W_{p}^{1}(\Gamma)} \leq K_{4}(p, I) .
$$

Let us consider the function $z(x, y)$, defined as

$$
z \stackrel{\text { def }}{=}(\omega+\psi) C_{\varepsilon}
$$

in the domain $\Omega_{1} \stackrel{\text { def }}{=}(0, \delta) \times(-1,2)$, where the functions $\psi$ and $C_{\varepsilon}$ are continued by periodicity into the extended region $\Omega_{1}$. The function $z$ is a solution to the following boundary value problem:

$$
\begin{aligned}
\Delta z & =2 \nabla \psi \nabla C_{\varepsilon} \equiv g, \text { in } \Omega_{1} \stackrel{\text { def }}{=}(0, \delta) \times(-1,2), \\
z & =0 \text { at } x=\delta, y \in(-1,2) \\
z & =0 \text { at } x=0, y \in(-1,2)
\end{aligned}
$$

Since

$$
g \in L^{r}\left(\Omega_{1}\right) \text { for any } 1<r<2
$$

and $z \in H^{1}\left(\Omega_{1}\right)$, applying the respective local estimates (see [8]) to the problem (91)(93), we obtain

$$
z \in W_{r}^{2}(\Omega) .
$$


Using the embedding Theorem (see [9]), we find that

$$
\frac{\partial z}{\partial x} \in L_{p}(\Gamma), p>1 \text {. }
$$

Substituting $x=\delta$ into the equality

$$
C_{\varepsilon} z_{x}=C_{\varepsilon x} C_{\varepsilon}(\omega+\psi)+\left(C_{\varepsilon y}+\psi_{x}\right) C_{\varepsilon}^{2},
$$

we obtain

$$
\left.\frac{1}{3} \frac{\partial}{\partial y}\left(C_{\varepsilon}^{3}\right)\right|_{x=\delta}=\left.\left.C_{\varepsilon}^{2}\right|_{x=\delta} C_{\varepsilon y}\right|_{x=\delta}=\left.\left.C_{\varepsilon}\right|_{x=\delta} z_{x}\right|_{x=\delta} \in L_{p}(\Gamma), p>1,
$$

uniformly in $\varepsilon>0$. The embedding of the space $W_{p}^{1}(\Gamma)$ into the space $C^{0, \alpha}(\bar{\Gamma})$ yields

$$
\left\|C_{\varepsilon}\right\|_{C^{0, \alpha}(\bar{\Gamma})} \leq K(I) \text { for } 0<\alpha<\frac{1}{3},
$$

uniformly in $\varepsilon$, and applying this estimate to the problem (69), (71), (72), we find, using $[8]$,

$$
\left\|C_{\varepsilon}\right\|_{C^{0, \alpha}(\bar{\Omega})} \leq K(I) \text { for } 0<\alpha<\frac{1}{3} .
$$

To prove the estimate (85), we consider an open set

$$
A^{+} \stackrel{\text { def }}{=}\left\{x=\delta, y: C_{\varepsilon}(\delta, y)>0\right\}
$$

on the boundary $\Gamma$, which consists of a countable number of open intervals, and a closed set

$$
A^{0} \stackrel{\text { def }}{=}\left\{x=\delta, y: C_{\varepsilon}(\delta, y)=0\right\}, \Gamma=A^{0} \cup A^{+} .
$$

Using the boundary condition (82), we obtain that

$$
C_{\varepsilon x}+f^{\varepsilon}=0, \text { for all } x=\delta, y \in A^{+}
$$

and applying the respective local estimates for the solutions of the elliptic boundary value problem, we deduce that $C_{\varepsilon}(x, y)$ is infinitely many times differentiable function in the vicinity of every point $(x, y) \in A^{+}$. Therefore, at every point $(x, y) \in A^{+}$, there exists a derivative $C_{\varepsilon x y}$ and

$$
\frac{\partial^{2} C_{\varepsilon}}{\partial x \partial y}+f_{\varepsilon}^{\prime}=0,(x, y) \in A^{+} .
$$

Using condition (82) we obtain that almost everywhere in the set $A^{0}$ there exists a derivative $C_{\varepsilon y}$ and

$$
\frac{\partial C_{\varepsilon}}{\partial y}=0,(x, y) \in A^{0} .
$$

Combining equations (96), (97), and (82), we deduce that the harmonic function $W \stackrel{\text { def }}{=}$ $C_{\varepsilon y} \in H^{1}(\Omega)$ and the following boundary condition holds:

$$
W=0,(x, y) \in A^{0}, \frac{\partial W}{\partial x}=-f^{\prime},(x, y) \in A^{+} .
$$

Multiplying the Laplace equation for the function $W$ by the function $\xi^{2} \max \{W-k, 0\}$, where $0 \leq \xi(x) \in C_{0}^{\infty}\left(A_{\rho}\right), A_{\rho}=\left\{x, y:\left(x-x_{0}\right)^{2}+(y-1)^{2}<\rho^{2}\right\}$ and $k$ is a positive 
constant, integrating it over the circle $A_{\rho}$, and applying integration by parts, we find that

$$
\begin{gathered}
\iint_{A_{k, \rho}} \xi^{2}|\nabla W|^{2} d x d y+2 \iint_{A_{k, \rho}} \xi(\nabla \xi \cdot \nabla W)(W-k) d x d y \\
+\int_{\Gamma_{k, \rho}} f^{\prime} \xi^{2} \max \{W-k, 0\} d y=0,
\end{gathered}
$$

where $A_{k, \rho} \stackrel{\text { def }}{=} A_{\rho} \cap\{W>k\} \cap \Omega$ and $\Gamma_{k, \rho}=A_{\rho} \cap\{W>k\} \cap \Gamma$. Rewriting the last integral in the equation (99) in the following form

$$
\int_{\Gamma_{k, \rho}} f^{\prime} \xi^{2} \max \{W-k, 0\} d x=2 \iint_{A_{k, \rho}} f^{\prime} \xi \xi_{x}(W-k) d x d y+\iint_{A_{k, \rho}} f^{\prime} \xi^{2} W_{x} d x d y
$$

we find that

$$
\iint_{A_{k, \rho}} \xi^{2}|\nabla W|^{2} d x d y \leq a_{0} \iint_{A_{k, \rho}}|\nabla \xi|^{2}(W-k)^{2} d x d y+a_{1} \operatorname{mes} A_{k, \rho},
$$

for some positive constants $a_{0}$ and $a_{1}$. The inequality (100) yields (see [8]) that

$$
W \in C^{0, \alpha}(\bar{\Omega}) \text { for some } \alpha>0
$$

and

$$
C_{\varepsilon} \in C^{1, \alpha}(\bar{\Omega})
$$

The norm of the function $C_{\varepsilon}$ in the space $C^{1, \alpha}(\bar{\Omega})$ depends on $\varepsilon>0$.

The decreasing (88) of the function $C_{\varepsilon}$ in $x$ follows immediately from the decreasing of the solution $C_{\varepsilon}^{\beta}$ in $x$ (see inequality (77)). To complete the proof we need to show that $W \geq 0$ in $\bar{\Omega}$. Applying the maximum principle we obtain that a negative minimum of $W$ may occur only on the part $A^{+}$of $\Gamma$. But the following inequality,

$$
\frac{\partial W}{\partial x}=-f^{\prime}(y) \geq 0
$$

holds on $A_{+}$and, using the Zaremba-Hopf-Giraud principle, we deduce that

$$
W=\frac{\partial}{\partial y} C_{\varepsilon} \geq 0 \text { in } \bar{\Omega}
$$

Corollary. The limiting function $C_{\varepsilon}$ is a unique solution of the following free boundary problem:

$$
\begin{gathered}
\Delta C_{\varepsilon}=0,(x, y) \in \Omega=(0, \delta) \times(0,1), \\
C_{\varepsilon}=0, \text { at } x=\delta \text { for } 0 \leq y \leq y_{0}(I) ; \frac{\partial}{\partial x} C_{\varepsilon}=-f \text { at } x=\delta \text { for } y_{0}(I) \leq y \leq 1, \\
C_{\varepsilon y}=0, \text { at } y=0, y=1, \\
C_{\varepsilon}=1, \text { at } x=0, \\
\int_{0}^{1} C_{\varepsilon}(\delta, y) d y=1-\frac{I \delta}{2} .
\end{gathered}
$$

The solutions $C_{\varepsilon}^{(\beta)}$ converge weakly in $H^{2}(\Omega)$ and strongly in

$$
W_{p}^{1}(\Omega) \cap W_{p}^{1}(\Gamma) \cap C^{0, \alpha}(\bar{\Omega}),(\text { for any } p>1, \alpha \in(0,1)),
$$


to the limiting solution $C_{\varepsilon}(x, y)$.

Proof. Using Theorem 4, we immediately find that the limiting function $C_{\varepsilon}$ is a solution to the problem (102)-(106). Let us prove the uniqueness of this solution. We assume, by way of contradiction, the existence of two different solutions $C_{1}$ and $C_{2}$ to the problem such that for $x=\delta$

$$
\begin{aligned}
& C_{1}=0,0 \leq y \leq y_{1}, C_{1}>0, y_{1}<y<1, \\
& C_{2}=0,0 \leq y \leq y_{2}, C_{2}>0, y_{2}<y<1,
\end{aligned}
$$

and $y_{1} \leq y_{2}$. Since for $x=\delta$ and $0 \leq y \leq y_{2}$ the difference $\bar{C}=C_{1}-C_{2} \geq 0$ and

$$
\frac{\partial \bar{C}}{\partial y}=0 \text { for } x=\delta, y_{2} \leq y \leq 1,
$$

using the maximum principle we obtain that $\bar{C} \geq 0$ in $\bar{\Omega}$. Then the free boundary condition (106) yields $\int_{0}^{1} \bar{C}(\delta, y) d y=0$ and $\bar{C}=0$ at $x=\delta$. Thus, $y_{1}=y_{2}$ and $C_{1}=C_{2}$ in $\bar{\Omega}$. Uniqueness of the limiting solution yields the convergence of the solutions $C_{\varepsilon}^{\beta}$.

THEOREM 5. The solutions $C^{\beta}$ of the problem (69)-(73) converge to the limiting solution $C(x, y)$ as $\beta \rightarrow 0$ weakly in the space $H^{1}(\Omega)$ and strongly in the space $L^{2}(\Omega)$. The function $C(x, y)$ is a unique solution of the following free boundary problem:

$$
\begin{gathered}
\Delta C=0,(x, y) \in \Omega=(0, \delta) \times(0,1), \\
C=0, \text { at } x=\delta \text { for } 0 \leq y \leq y_{0}(I) ; \frac{\partial}{\partial x} C=-f \text { at } x=\delta \text { for } y_{0}(I) \leq y \leq 1, \\
C_{y}=0, \text { at } y=0, y=1, \\
C=1, \text { at } x=0, \\
\int_{0}^{1} C(\delta, y) d y=1-\frac{I \delta}{2} .
\end{gathered}
$$

It decreases in $x$, increases in $y$ and belongs to the space $C^{0, \alpha}(\bar{\Omega}), 0<\alpha<1 / 3$.

Proof. The solutions $C_{\varepsilon}^{\beta}$ are bounded in the space $H^{1}(\Omega)$ uniformly in $\varepsilon>0$ and $\beta>0$ and converge to the solution $C^{\beta}$ as $\varepsilon \rightarrow 0$ weakly in the space $H^{1}(\Omega)$ and strongly in the space $L^{2}(\Omega)$. They converge to the limiting solutions $C_{\varepsilon}$ as $\beta \rightarrow 0$ weakly in the space $H^{1}(\Omega)$ and strongly in the space $L^{2}(\Omega)$ uniformly in $\varepsilon>0$. Finally, the limiting solutions $C_{\varepsilon}$ converge to the solution $C$ as $\varepsilon \rightarrow 0$. All of these convergences yield the convergence of the solutions $C^{\beta}$ to the solution $C$ as $\beta \rightarrow 0$ weakly in the space $H^{1}(\Omega)$ and strongly in the space $L^{2}(\Omega)$.

Monotonicity in $x$ and $y$ of the solution $C$ follows from the inequalities (86), (87). Finally, the uniform in $\varepsilon>0$ estimate (94) yields the Hölder continuity of the limiting solution $C(x, y)$ with exponent $\alpha$, for every $\alpha \in(0,1 / 3)$.

\section{REFERENCES}

[1] I. Rubinstein and B. Zaltzman, Electro-osmotically induced convection at a permselective membrane, Physical Review E, 62, pp. 2238-2251, 2000.

[2] I. Rubinstein and B. Zaltzman, Electroosmotic slip of the second kind and instability in concentration polarization, Mathematical Models and Methods in Applied Sciences, 11 (2), pp. 263-300, 2001. 
[3] I. Rubinstein, B. Zaltzman, J. Pretz, and C. Linder, Experimental verification of the electroosmotic mechanism of overlimiting conductance through a cation exchange electrodialysis membrane, Russian Electrochemistry 38, pp. 853-864, 2002.

[4] I. Rubinstein, B. Zaltzman and T. Pundik, Ion-Exchange Funneling in Thin Film Coating Modification of Heterogeneous Electrodialysis Membrane, Phys. Rev. E, 65, art. number 041507, 2002.

[5] C. Linder, G. Saveliev, Y. Mirsky, and O. Kedem, in ICOM2000, Kibbutz Ma'ale Hachamisha, Hills of Jerusalem, Israel, September 24-27, 2000, Abstracts, pp. 104-105.

[6] I. Rubinstein, Electrodiffusion of Ions, SIAM, Philadelphia, p. 149, p. 109, p. 170, 1990.

[7] D. Gilbarg and N. S. Trudinger, Elliptic partial differential equations of second order. Reprint of the 1998 edition. Classics in Mathematics. Springer-Verlag, Berlin, p. 517, 2001.

[8] O. A. Ladyzhenskaya and N. N. Ural'tseva, Linear and quasilinear elliptic equations. (Translated from the Russian) Academic Press, New York-London, p. 495, 1968.

[9] S. M. Nikol'skij, Approximation of functions of several variables and imbedding theorems. (Translated from the Russian) Springer-Verlag, New York-Heidelberg, p. 418, 1975. 